\title{
Arthroscopic Revision Surgery of Failed Open Bankart Repair: Case Reports
}

\author{
Sedeek Mohamed Sedeek ${ }^{\mathrm{a}, \mathrm{b}}$, Tan Hwee Chye Andrew ${ }^{\mathrm{a}}$
}

\begin{abstract}
The clinical outcome of revision surgery for the failed Bankart repair is not well known. The purpose of this case study is to report the success rates achieved in two cases using arthroscopic techniques to revise failed open Bankart repairs. Both patients showed significant improvements in terms of shoulder stability and function at post-arthroscopic revision with no recurrences on follow-up. Their overall function and stability were evidenced by the improvements in shoulder scores and subjective scoring. In conclusion, arthroscopic revision of failed open anterior shoulder stabilisation using suture anchors is a reliable procedure in relation to the recurrence rate, range of motion and shoulder function in carefully selected patients, and with a proper workup.
\end{abstract}

Keywords: Shoulder; Anterior instability; Arthroscopy; Revision; Bankart repair

\section{Introduction}

Traumatic anterior shoulder instability is the most common type of glenohumeral instability. The pathology, treatment and results of shoulder instability have been well documented in the literature [1]. However, persistent or recurrent glenohumeral instability after a previous operative stabilization represents an uncommon and complex problem for both the patient and the treating physician [2].

The reasons for failure after anterior stabilization have been reported to include bony glenoid defects, engaging Hill-Sachs lesions, tear of the lateral capsule and traumatic

\footnotetext{
Manuscript accepted for publication June 6, 2013

aSports Medicine Service, Department of Orthopaedic Surgery, Singapore General Hospital, Outram Road 169608, Singapore ${ }^{\mathrm{b}}$ Corresponding author: Sedeek Mohamed Sedeek, Department of Orthopaedic Surgery, Singapore General Hospital, Outram Road 169608, Singapore. Email: sedeeko2000@hotmail.com

doi: http://dx.doi.org/10.4021/jmc1354w
}

events [3]. Treatment options for failed shoulder instability repair include conservative care with physical therapy, bracing and activity modifications, and revision of surgical intervention [4].

Typically, revision surgery in these cases has been undertaken by an open approach, although arthroscopic revision appears to be technically possible in many cases and hypothetically provides potential for an expedited recovery owing to less surgical morbidity [5]. Whether the results of initial arthroscopic stabilization differ from those of arthroscopic revision surgery is still not entirely clarified [6].

We present two cases of failed open Bankart repair that were treated by arthroscopic revision shoulder stabilization. We aimed to study and report the outcomes of arthroscopic revision surgery following failed open Bankart repair in view of the limited available literature.

\section{Case Report}

\section{Case 1}

Our first patient is a 16-year-old student who fell down and sustained a traumatic right anterior shoulder dislocation in 2001. The first attack was self-reduced. However, subsequently, the patient complained of recurrent right-shoulder dislocations associated with pain.

A magnetic resonance imagery (MRI) scan of the right shoulder revealed a soft tissue Bankart lesion with no bony involvement or Hill-Sachs lesion. The first open Bankart repair was done in January 2002. Operative findings were that of a soft tissue Bankart lesion at the 5 o'clock position associated with capsular laxity. Open Bankart repair with three absorbable anchor sutures and inferior capsular shift were performed.

Approximately 2 months later, the patient had a nontraumatic dislocation of the shoulder. He was lifting his arm overhead to reach for something on the shelf when he dislocated his shoulder. Subsequently, he had frequent dislocations which were self-reduced.

At this stage, the patient presented to our clinic. On examination, he had a positive apprehension test; forward el- 
evation and abduction were up to 110 degrees; and external rotation was up to 65 degrees. There was no vascular or neurological abnormality in the limb.

Multiple x-rays were done that did not show any bony lesion; while, the MRI revealed a large Bankart lesion associated with capsular laxity.

We decided to do an arthroscopic revision Bankart repair for him. Various aspects of the shoulder before and after surgery were compared in the patient using simple shoulder test (SST) [7] and the University of California at Los Angeles (UCLA) scoring system [8].

Arthroscopic revision surgery was performed in October 2004, about 2 years' post-open-surgery. Intra-operative findings were that of a large Bankart lesion from 2 o'clock to 6 o'clock associated with anterior inferior capsular laxity. After noting the above findings on arthroscopy, arthroscopic repair ensued. The Bankart lesion was repaired using three suture anchors, and the anterior inferior laxity was corrected using a pinch-tuck technique [9] to plicate the paralabral capsule.

On follow up, the patient had a better range of movement and significant improvement in aspects of the SST and UCLA scoring system [7, 8]. At the latest follow up 3 years postoperatively, the active range of motion measured 160 degrees in forward elevation and 150 degrees abduction, with an external rotation of 80 degrees. The patient was free from re-dislocation. At the same time, the functional outcome was assessed. The UCLA shoulder scores assessed levels of pain, function and range, and strength of forward flexion as well as satisfaction of the patient. The scores improved from 16 to 30 . The SST score showed improvements in terms of an increase of yes responses in four areas. Additionally, the patient resumed his former duties and sport activities with greater ease and comfort.

\section{Case 2}

Our second patient is a 38-year-old gentleman who works as a porter at the airport. His first episode of shoulder dislocation occurred in July 1991 while he was playing squash. He was placing his arm in the overhead position while trying to hit the ball when the dislocation occurred. The dislocation was self-reduced. Subsequently, he complained of pain when carrying heavy loads and this affected his work. He had two more episodes of dislocation before being seen by the orthopaedic department.

Open Bankart repair surgery was done in May 1992. Intraoperative findings were that of a moderate Bankart lesion with inferior capsular laxity. No bony lesion was detected. The operation was done through a Henry's approach with dissection of the deltopectoral groove and division of the subscapularis. The Bankart lesion was repaired with the open Bankart repair done with sutures through intra-osseus tunnels. Inferior capsular laxity was corrected with capsular

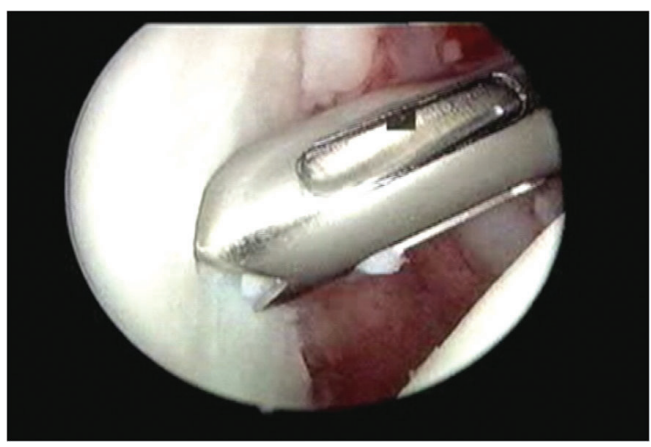

Figure 1. The drill hole for the anchor was placed on the glenoid face by $3 \mathrm{~mm}$ onto the articular surface.

shift technique.

The patient subsequently returned to work. About 1 year later, the patient fell onto his right shoulder and dislocated it. It was self-reduced. Open revision surgery was then performed in November 1994. The findings were a 3 o'clock Bankart lesion, and subcapsular laxity of the right glenohumeral joint capsule. Putti Platt repair was done through Henry's approach with double-breasted stitches to correct the laxity.

Three years later, patient had an episode of right shoulder dislocation again. However, in view of the two previous open surgeries which failed, the patient was given conservative management. His symptoms persisted and dislocation frequency was once every 2 weeks. He was able to self-reduce the dislocations but they did limit his work abilities.

In 2006, an MRI scan of the right shoulder showed the anterior glenoid to be deficient on a short strip of anterior periosteum. There was a defect at the posterolateral margin of the humeral head in keeping with a Hill-Sachs lesion. The subscapularis was waxy and lax, and the inferior glenohumeral ligament appeared to be attenuated.

In January 2006, arthroscopic revision surgery was performed. Intraoperative arthroscopic findings were that of a right shoulder Bankart lesion from the 3 o'clock to the 6 o'clock position associated with a small Hill-Sachs lesion. No capsular laxity was noted. Articular surfaces were observed to be intact. Arthroscopic repair of the identified Bankart lesion was done with three suture anchors.

The patient was then followed up for 30 months. He did not have any dislocations; the apprehension test was negative; and the external rotation was at 70 degrees. His UCLA shoulder scores improved from 17 to 32 . The simple shoulder test showed improvements in seven areas. He is now able to carry out his work duties with greater ease and comfort, requiring less help from his colleagues.

The arthroscopic revision surgeries were performed by the same surgeon with the use of a standardised technique in both patients. After induction of a general anaesthesia, the patient was placed in a beach-chair position and a thorough 


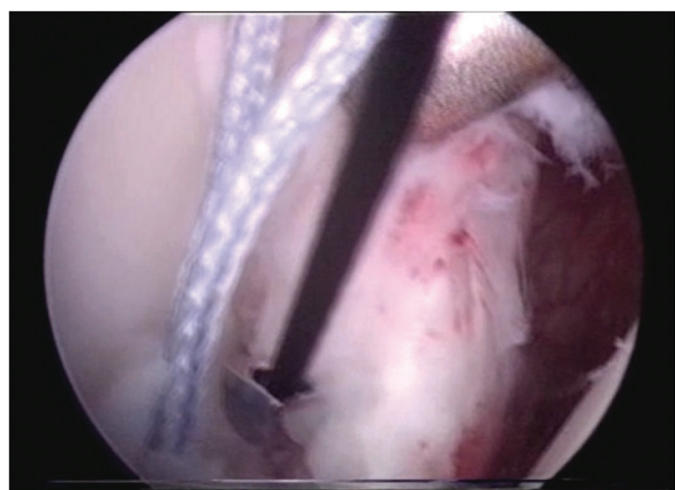

Figure 2. The suture passer was passed under the Bankart lesion.

examination under anaesthesia was performed to assess the magnitude and direction of instability. The shoulder was prepared and draped in a sterile manner, and the bony landmarks were marked carefully to maintain orientation throughout the procedure. A standard posterior viewing portal was established approximately $2 \mathrm{~cm}$ inferior and $1 \mathrm{~cm}$ medial to the acromial angle. Two anterior portals were established using the outside-in technique with a spinal needle to establish the most appropriate placement of the cannulas. The anterosuperior portal was made in the rotator interval just inferior to the anterior edge of the acromion, and the anterior midglenoid portal was made just over the superior border of the subscapularis tendon.

A small cannula was inserted into the anterosuperior portal, and a large-diameter threaded cannula was placed in the anterior mid-glenoid portal. Complete diagnostic arthroscopy was done through the posterior and anterior portals, with assessment of the glenoid labrum, capsule, rotator cuff and the humeral head for possible Hill-Sachs lesions.

The Bankart lesion was mobilised from the anterior glenoid surface using a periosteal elevator. The Bankart lesion must be completely freed from the neck of the glenoid and at the completion of this step, the subscapularis muscle can be clearly visible through the tear site. The goal was to mobilise the labrum so it could be shifted superiorly and laterally. The glenoid neck was lightly abraded using a rasper. The first anchor was placed at the 5: 30 clock position, on the glenoid articular surface $3 \mathrm{~mm}$ from the articular edge (Fig. 1). We believe this is essential in recreating the labral bumper, and in re-establishing the concavity compression effect. The most inferior placement would ideally be placed at the 6 o'clock position; however, this is often not possible due to limitations in the placement angle.

A suture passer was passed under the Bankart lesion (Fig. 2). The suture strand of the suture anchor nearer the labrum was brought out through the anterosuperior portal, and in turn through the labrum in a retrograde fashion using the suture passer, and retrieved from the mid-glenoid portal.

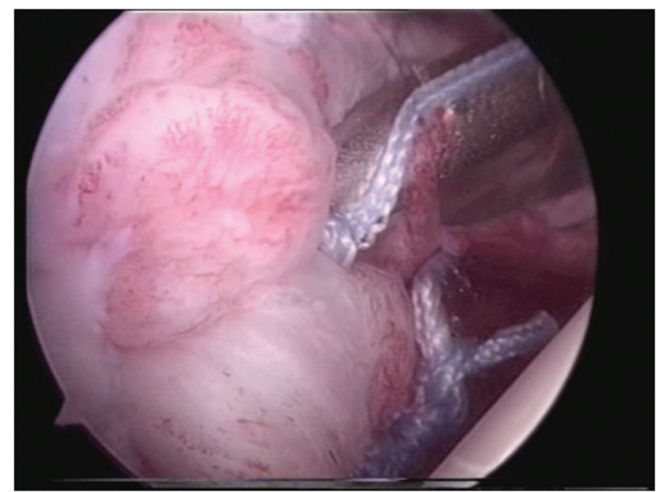

Figure 3. The knot rest on the capsular side on the gleniod labrum not on the articular side.

This suture limb remained as the post during suture tying and this would ensure that the knot rest on the capsular side of the glenoid labrum and not on the articular side (Fig. 3). This technique would effectively push the labrum up towards the glenoid socket, thereby recreating the labral bumper. The sutures were tied using the Tennessee slider knot, which is easy to tie, has a low profile and possesses good holding strength [10-12]. The second and third suture anchors were done at the 4:30 and 3:30 clock positions in the same manner. The anteroinferior capsular laxity was managed in the first patient by the pinch tuck technique [9]; the suture passer would be passed through the perilabral capsule $1 \mathrm{~cm}$ anterior and $1 \mathrm{~cm}$ inferior to the Bankart lesion to plicate the redundant capsule.

Postoperatively, in both cases, the patient's relevant arm was placed in a sling for 6 weeks. They were allowed to do pendular motion exercises for the first 3 weeks, followed by elevating the elbow to shoulder level (forward active flexion to 90 degrees) from the third to the sixth week. They were also taught to do isometric rotator cuff exercises during these 6 weeks. Full shoulder mobilisation was allowed after 8 weeks. Sport activities were allowed at 6 months.

\section{Discussion}

The Bankart repair is considered the standard, primary surgical treatment for anterior shoulder instability. Although this procedure is successful, failures can still occur [13].

Initial arthroscopic stabilization has been shown to yield comparable results to open procedures. Nonetheless, only some authors have evaluated the outcome the arthroscopic salvage or stabilization techniques [6].

Kim et al. [9] analysed a series of 23 patients that were arthroscopically re-stabilized with the use of suture anchors for failed primary Bankart repair. Recurrence developed in five patients after revision surgery. Good or excellent results were found in 19 patients, while a return to the preopera- 
tive level of activity was achieved in 18 patients. Neri et al. [5] followed up with 11 patients after arthroscopic revision Bankart repair. Eight patients showed good to excellent results, whilst subluxation or dislocation occurred in three cases.

Recurrent instability after a primary stabilization procedure would be considered a complex situation, as it represents a diagnostic as well as surgical challenge to re-establish the shoulder despite scarring and anatomic distortion from a previous procedure [14].

Generally, trauma, diagnostic errors and technical errors are the three major causes that can lead to failure of anterior stabilization procedures. However, initial misdiagnoses of concomitant pathology have constituted $85 \%$ of the causes of recurrent glenohumeral instability after a previous surgical stabilization $[13,15]$.

Therefore, the assessment of a failed shoulder instability procedure begins with an appropriate diagnosis and determination of the mode of failure. Thus, a through history is a crucial factor. Several key issues in the patient's history are important including a potential mechanism of re-injury, previous surgical treatment of the shoulder and whether this stabilization ever relieved the symptoms of the patient [1].

In conjunction with the history, physical examination is essential to define the direction and magnitude of the instability; assess range of motion and pain; and assess co-existing, previously untreated pathology. The structure, function, neurological status and strength of the injured shoulder should be examined and compared with the contralateral shoulder [1].

Appropriate imaging can serve as an adjunct to successful evaluation and diagnosis of bone loss and concomitant pathology in patients with recurrent instability. Preoperative radiographs include the anteroposterior, scapular-Y and axillary views. Additionally, a Stryker notch view is helpful for evaluating Hill-Sachs lesions. Nevertheless, it should be emphasised that radiographic examinations are not always easy to interpret because of artifacts from scarring and hardware from the previous surgical procedures [16]. A computerised tomography scan is a useful way to determine the extent of any bone loss in the humeral head or glenoid component. MRI is the modality of choice to evaluate the glenoid labrum and glenohumeral ligament complex [1].

In contrast to open repairs, arthroscopy allows the inspection of the whole joint and optimal treatment of additional SLAP lesions, the repair of posterior capsulolabral lesions, and inferior plications. In addition, open revision of primary open repair could encounter some difficulties in these particular cases because of the scarring between the capsule and the subscapularis [3, 17]. This could lead to a higher rate of capsular over-tensioning with loss of external rotation and subscapularis muscle insufficiency $[3,17]$.

In our case reports, both patients showed significant improvements in terms of shoulder stability and function at post-arthroscopic revision with no recurrences on followup. Their overall function and stability improvements were evidenced by the improvements in the shoulder scores and subjective scoring. Traditionally, failed shoulder instability repair has been addressed with open procedures with many variations of capsular shifts [18]. However, both our patients had previous open surgeries and did well post-arthroscopic revision. Moreover, in the second patient the arthroscopic stabilization was done after two failed open surgeries.

We believe that there are some important technical points that should be utilized in the arthroscopic revision techniques. Firstly, the Bankart lesion should be completely mobilised. This dissection could be tedious; however, every attempt should be made to avoid thinning or harming the glenohumeral ligament during the dissection. Secondly, there must be sufficient re-tensioning of the capsulolabral complex and adequate incorporation of the inferior glenohumeral ligament, which is the main restraint against anterior translation in abducted and external rotation position [3]. We place the suture anchors on the articular surface $3 \mathrm{~mm}$ from the glenoid edge to ensure re-creation of the bumper effect and re-tensioning of the inferior glenohumeral ligament. Thirdly, we use at least three suture anchors placed at the clock positions of 3:30, 4:30, and 5:30 as a sufficient number of sutures is necessary for successful arthroscopic repair of the instability [9]. Additionally, the surgical procedure of the arthroscopic revision Bankart repair using suture anchors has variable difficulties depending on the type of primary reconstructive procedure [19]. Patients with suture repair without hardware present few obstacles for the revision surgery. However, in patients with previous metallic suture anchors, the insertion of new anchors can be difficult. Close observation and viewing from different portals can eliminate the possible complications related to the anchor placement, such as the breakage of the drill tip or bone punch [9]. Fourthly, cases of capsular laxity should be managed arthroscopically and meticulously as unaddressed anteroinferior capsular laxity could lead to the failure of the arthroscopic stabilization. Lastly, our contraindication for the arthroscopic revision technique are voluntary dislocation, significant or engaging Hill-Sachs lesions, severe erosion of the glenoid rim or inverted pear glenoid as described by Burkhart and De Beer [20], severe osteoarthritis and shoulder stiffness [16].

\section{Conclusion}

Arthroscopic revision of failed open anterior shoulder stabilization using suture anchors is a reliable procedure in relation to recurrence rate, range of motion and shoulder function in carefully selected patients with a proper work-up.

\section{Competing Interests}


All authors have no competing interests in this report.

\section{References}

1. Ghodadra N, Grumet R, LeClere L, Matthew L. Failed shoulder stabilization surgery. Current Orthopaedic Practise. 2009;20(4)365-373.

2. Meehan RE, Petersen SA. Results and factors affecting outcome of revision surgery for shoulder instability. J Shoulder Elbow Surg. 2005;14(1):31-37.

3. Bartl C, Schumann K, Paul J, Vogt S, Imhoff AB. Arthroscopic capsulolabral revision repair for recurrent anterior shoulder instability. Am J Sports Med. 2011;39(3):511-518.

4. Creighton RA, Romeo AA, Brown FM, Jr., Hayden JK, Verma NN. Revision arthroscopic shoulder instability repair. Arthroscopy. 2007;23(7):703-709.

5. Neri BR, Tuckman DV, Bravman JT, Yim D, Sahajpal DT, Rokito AS. Arthroscopic revision of Bankart repair. J Shoulder Elbow Surg. 2007;16(4):419-424.

6. Krueger D, Kraus N, Pauly S, Chen J, Scheibel M. Subjective and objective outcome after revision arthroscopic stabilization for recurrent anterior instability versus initial shoulder stabilization. Am J Sports Med. 2011;39(1):71-77.

7. Lippitt SB, H, Masten FA. A practical tool for evaluating function: The simple shoulder test. In: Masten FA, Fu FH, Hawkins RJ, eds. The Shoulder: A Balance of Mobility and Stability. Rosemont, IL: American Academy of Orthopaedic Surgeons, 1993:501-518.

8. Ellman H, Hanker G, Bayer M. Repair of the rotator cuff. End-result study of factors influencing reconstruction. J Bone Joint Surg Am. 1986;68(8):1136-1144.

9. Kim SH, Ha KI, Kim YM. Arthroscopic revision Bankart repair: a prospective outcome study. Arthroscopy. 2002;18(5):469-482.

10. McMillan ER, Caspari RB. Arthroscopic knot-tying techniques. In: Imhoff AB, Ticker JB, Fu FH, eds. At- las of Shoulder Arthroscopy. London: Martin Dunitz, 2003:81-95.

11. Nottage WM. Sutures, anchors, and knots. Presented at the 18th annual meeting. Fall course, Arthroscopy Association of North America, Vancouver, BC, Canada, April 1999.

12. Baumgarten KM, Wright RW. Ease of tying arthroscopic knots. J Shoulder Elbow Surg. 2007;16(4):438-442.

13. Cho NS, Yi JW, Lee BG, Rhee YG. Revision open Bankart surgery after arthroscopic repair for traumatic anterior shoulder instability. Am J Sports Med. 2009;37(11):2158-2164.

14. Marquardt B, Garmann S, Schulte T, Witt KA, Steinbeck J, Potzl W. Outcome after failed traumatic anterior shoulder instability repair with and without surgical revision. J Shoulder Elbow Surg. 2007;16(6):742-747.

15. Boileau P, Villalba M, Hery JY, Balg F, Ahrens P, Neyton L. Risk factors for recurrence of shoulder instability after arthroscopic Bankart repair. J Bone Joint Surg Am. 2006;88(8):1755-1763.

16. Boileau P, Richou J, Lisai A, Chuinard C, Bicknell RT. The role of arthroscopy in revision of failed open anterior stabilization of the shoulder. Arthroscopy. 2009;25(10):1075-1084.

17. Levine WN, Arroyo JS, Pollock RG, Flatow EL, Bigliani LU. Open revision stabilization surgery for recurrent anterior glenohumeral instability. Am J Sports Med. 2000;28(2):156-160.

18. Young DC, Rockwood CA, Jr. Complications of a failed Bristow procedure and their management. J Bone Joint Surg Am. 1991;73(7):969-981.

19. Robinson CM, Howes J, Murdoch H, Will E, Graham C. Functional outcome and risk of recurrent instability after primary traumatic anterior shoulder dislocation in young patients. J Bone Joint Surg Am. 2006;88(11):2326-2336.

20. Burkhart SS, Cole BJ. Bridging self-reinforcing doublerow rotator cuff repair: we really are doing better. Arthroscopy. 2010;26(5):677-680. 